\title{
Léon-Gontran Damas. Cent ans en noir et blanc, éd.
} Antonella Emina

\section{Elena Fermi}

\section{(2) OpenEdition}

1 Journals

\section{Édition électronique}

URL : http://journals.openedition.org/studifrancesi/5456

DOI : 10.4000/studifrancesi.5456

ISSN : 2421-5856

Éditeur

Rosenberg \& Sellier

\section{Édition imprimée}

Date de publication : 1 décembre 2016

Pagination : $574-576$

ISSN : 0039-2944

\section{Référence électronique}

Elena Fermi, « Léon-Gontran Damas. Cent ans en noir et blanc, éd. Antonella Emina », Studi Francesi [En ligne], 180 (LX | III) | 2016, mis en ligne le 01 janvier 2017, consulté le 18 septembre 2020. URL : http:// journals.openedition.org/studifrancesi/5456; DOI : https://doi.org/10.4000/studifrancesi.5456

Ce document a été généré automatiquement le 18 septembre 2020.

\section{(c) $($ ) $(9)$}

Studi Francesi è distribuita con Licenza Creative Commons Attribuzione - Non commerciale - Non opere derivate 4.0 Internazionale. 


\title{
Léon-Gontran Damas. Cent ans en noir et blanc, éd. Antonella Emina
}

\author{
Elena Fermi
}

\section{RÉFÉRENCE}

Léon-Gontran Damas. Cent ans en noir et blanc, sous la direction de Antonella EMINA, Paris, CNRS Éditions, 2014, 340 pp.

1 Léon-Gontran Damas. Cent ans en noir et blanc, produit de l'effort combiné de spécialistes de renommée internationale, est le résultat d'un projet concernant l'ensemble de l'œuvre de l'écrivain guyanais, fondateur, avec Aimé Césaire et Léopold Sédar Senghor, du mouvement de la Négritude, dont il représente une figure charnière. L'objectif des chercheurs ayant contribué aux études qui y sont présentées est celui d'interroger et d'analyser à la fois les écrits damassiens - dont la portée n'a pas toujours été relevée par la communauté des littéraires et des spécialistes de la francophonie - et la figure de Damas lui-même. Le volume, divisé en quatre sections, réunit quatorze études conduites par treize auteurs ayant des formations et des spécialisations différentes. Parmi eux, deux écrivains guadeloupéens et un poète et écrivain nigérien: Maryse CONDÉ, Daniel MAXIMIN et Femi OJO-ADE. La composition de l'équipe a pour but de garantir une perspective multifocale qui permette de rendre compte des multiples facettes caractérisant l'œuvre et la figure de Damas.

2 La première section, «Des traces, des tracés», rassemble trois études de Daniel MAXIMIN, Femi OJO-ADE et Maryse CONDÉ, ce qui lui donne sa marque distinctive. Le point de vue qui y est mis en avant est en effet celui de trois écrivains, trois «collègues» de Damas en un sens, qui vont donc évoquer sa figure plutôt selon une perspective littéraire, créative et artistique qu'analytique et universitaire.

Daniel MAXIMIN annonce le plan de son étude, Léon Damas, Étoile pigmentée de graffiti... en l'introduisant avec une citation poétique, en guise d'exergue, tirée du recueil Black Label (1956). Son article, ponctué des vers damassiens, parcourt la biographie de l'écrivain 
guyanais, en soulignant son positionnement au carrefour de trois continents: l'Amérique où il est né, l'Afrique de ses origines et l'Europe où il a reçu sa formation universitaire et où il a rencontré ses deux compagnons dans la Négritude, Césaire et Senghor. Trois continents qui semblent correspondre aux trois disparitions féminines qui ont marqué dès le début la vie du poète et qui confèrent ainsi à son œuvre une empreinte particulière de solitude: la mort de sa sœur jumelle et les décès prématurés de sa mère et de sa grand-mère qui s'unissent à l'abandon par son père. La poésie deviendrait ainsi chez Damas à la fois une manière de combler le vide que les événements de sa vie ont laissé en lui et une forme de combat. Colonisé débarqué chez les colonisateurs, il dénonce à sa façon la colonisation, les préjugés raciaux, l'exil inhospitalier, l'acculturation, mais aussi la soumission, le mimétisme, les fauxsemblants, l'aliénation complaisante, les masques blancs de lui-même et des siens. Dès le début, sa vie et son œuvre vont se caractériser par le fait d'être «contre», de ne pas trouver d'excuses, ni aux autres, ni à elles -mêmes. Un choix tragique qui toutefois ne se résout pas en une capitulation. La poésie et l'écriture ponctuent la vie de Damas et nourrissent son combat qui se caractérise par la fidélité à sa Guyane natale, aux Amériques du Nord et du Sud en passant par la Caraïbe, aux poètes de tous bords, à la poésie comme parole première, fondatrice et nécessaire, à lui-même et à l'écriture de son destin.

Femi OJO-ADE, dans son étude Un solitaire au sein d'une solidarité: la vie de Léon-Gontran Damas, évoque le souvenir de sa première et unique rencontre avec le guyanais au printemps 1974 à l'université de Toronto. Après avoir rapidement parcouru l'enfance et la jeunesse de l'intellectuel jusqu'à son arrivée à Paris et à la rencontre avec Césaire et Senghor, l'auteur se concentre sur la position politique de Damas dans le trio de la Négritude. Mulâtre entre deux noirs, il se distinguait d'eux par la couleur de sa peau, tout en partageant avec eux une petite taille. Sa carrière politique a été brève et n'a pas vraiment marqué sa biographie, tandis que ses compagnons de combat ont fait de l'engagement politique un des objectifs de leur vie, en faisant connaitre au monde entier les enjeux de la colonisation et de la décolonisation en Afrique et dans la Caraïbe. Selon le critique, Damas est en revanche resté toujours contre, en refusant les compromis que le jeu politique impose et a continué à mener son combat en solitaire. Sorti bientôt de la politique il est vite revenu à l'écriture, seule forme de lutte qui le laissait totalement libre et qui, de ce fait, lui correspondait davantage. Seul parmi les pères de la Négritude, il est resté toute sa vie en exil, ce qui lui a permis de mieux comprendre le maître, donc de mieux le combattre. Il a mené une vie de marronnage, assumant totalement sa liberté, même dans ses côtés les plus déchirants.

5 Maryse CONDÉ, dans Léon-Gontran Damas aux USA, fait partager aux lecteurs sa première expérience d'enseignement aux États-Unis, à l'Université de Santa Barbara en Californie où, après avoir proposé sans succès Césaire puis Senghor, elle décida de se pencher sur Damas qu'elle connaissait à peine mais qui suscita, de manière inattendue, la passion de ses jeunes étudiants, car sa poésie permettait à chacun de la lire à sa manière et d'en donner une interprétation personnelle.

La deuxième section, «Des poèmes, de la poésie», se penche sur l'écriture poétique de Damas. L'étude de Femi oJo-ADE qui ouvre cette partie du volume, est entièrement dédiée à l'analyse du poème «Hoquet», extrait du recueil Pigments (1937). L'auteur considère en effet que ce poème se distingue de tous les autres présents dans le recueil, car c'est le seul qui permet aux lecteurs de contempler l'expérience vécue et les 
opinions de Damas. On y retrouve les sujets principaux de l'écriture damassienne, la solitude, la lutte sans répit pour la conquête de sa propre liberté contre la soumission au joug du colonisateur, la tragédie toujours en passe d'éclater, traités avec un style qui rappelle le rythme populaire africain. Ojo-Adé conclut son analyse en ouvrant un volet sur les définitions de Négritude et Créolité. Le style de sa poésie rapproche Damas de ses racines créoles, qu'il partage avec Césaire et le ferait donc s'inscrire parmi les écrivains dits «de la créolité». L'auteur remet ici en question les deux définitions, celle de Négritude et celle de Créolité, en en montrant les limites. Si la catégorisation peut être utile pour les chercheurs, elle a le tort de vouloir forcer des intellectuels de grande envergure tels que Senghor, Césaire ou Damas dans des bornes qui ne leur appartiennent pas. Damas notamment se distinguerait des deux autres pour son rejet de toute compromission, pour l'acceptation de ses racines diasporiques.

Kathleen GYSSELS s'interroge elle aussi sur la poésie damassienne dans son étude intitulée «Désirs comprimés d'un bel enfant de chœur». L'entre-dit genré. Elle décide de relire le recueil Black-Label à l'aide de l'ensemble de l'œuvre de Damas afin «d'y dénicher, [..], l'anxiété masculine noire, ainsi que le tabou de l'amour interracial (p. 76)». À travers l'analyse stylistique et linguistique des poèmes, l'auteur décèle quelques caractéristiques fondamentales de l'œuvre du guyanais: le démantèlement du «Je» stable et autoritaire au profit d'une identité plurielle et changeante, l'instrumentalisation du corps noir au service des plaisirs et divertissements des Blancs, la protection de l'identité sexuelle qui déguise le genre de l'amour, un sémantisme corporel qui cache l'oppression par les Français, les processus de l'assimilation et du blanchiment, l'expression d'un malaise irrésolu. Le français standardisé se réinvente chez Damas afin de dire l'indicible.

La contribution d'Isabella Maria zopPI, Deux langues, deux voix, un texte: Damas lit Langston Hughes, clôt cette section consacrée au domaine poétique. Le chercheur y conduit une étude comparée entre deux langues et deux voix: celles, francophones, de Damas et celles, anglophones, de Langston Hughes. En examinant la biographie des deux écrivains, ponctuée de rencontres au fil des années, l'auteur développe la relation idéologique et langagière entre les deux intellectuels à l'aide du poème de Hughes «Let America be America again» (1935) et de la traduction qu'en fit Damas. Vers après vers, Zoppi développe une analyse ponctuelle de la traduction damassienne qui se révèle être une véritable réécriture, où Damas fait ressortir sa propre vision du message porté par Hughes, sa personnelle interprétation.

Un essai de Marie-Christine HAZAËL-MASSIEUX, intitulé Damas et ses langues, le français et le créole ou l'interdit du fruit défendu, ouvre la troisième section du volume «Des mots, des signes», consacrée à l'observation et à l'analyse de la langue damassienne dans l'ensemble de son œuvre, en particulier aux interférences qu'on y trouve des créoles (guyanais et martiniquais particulièrement) et à l'utilisation poétique du français. Exemples tirés de l'œuvre à l'appui, l'auteur réfléchit sur la relation toujours difficile et jamais innocente entre le créole et le français, qui cohabitent nécessairement dans l'œuvre du guyanais. Leur relation se révèle être toujours déséquilibrée en faveur du français, dont la richesse et le prestige ont permis à Damas de faire sa carrière en France et dans le monde, tandis que le créole est toujours considéré comme un patois, même si dans l'œuvre damassienne les influences du créole et de la culture guyanaise sont évidentes. Au bout du compte, la langue de Damas révèle une fécondité visuelle et musicale qui fait du guyanais un proche du surréalisme et un grand poète francophone. 
10 Marco MODENESI et Jacques CHEVRIER consacrent leurs articles à Veillées noires, recueil de contes dérivant du matériel rapporté de la mission que le jeune Damas avait entreprise en Guyane en 1934. Modenesi se met À l'écoute des "Veillées noires", dans la mesure où il concentre son attention sur la dimension orale de ces textes. Comme chez la plupart des écrivains francophones, pour Damas aussi l'oralité cohabite avec l'écriture, faisant partager aux contes guyanais du recueil «l'univers de la parole d'une civilisation où l'écriture a des rôles différents de la création artistique» (p.160). La dimension du conte se révèle être, chez Damas, bien plus qu'une simple redécouverte de ses racines guyanaises. Il s'agit bien au contraire de sauvegarder, à travers la transcription de ces textes, un héritage ancestral qui pourra être mis à la disposition d'autres générations et d'autres cultures, car Damas, loin de s'adresser seulement aux lecteurs guyanais, vise le public de l'Europe et de l'Occident, auquel il veut faire redécouvrir une culture que la société occidentale a oubliée, sinon véritablement perdue. CHEVRIER, dans son étude "Veillées noires". Le "coeur-de-chauffe» de Léon-Gontran Damas?, se consacre à l'analyse de ce recueil de contes où l'écrivain s'éloigne de l'Afrique des origines, invoquée dans Pigments, pour se replonger dans l'univers créole de son enfance. En explorant le recueil, Chevrier arrive à affirmer qu'il constituerait, dans l'œuvre damassienne, un véritable manifeste de la culture guyanaise, caractérisé par une double stratégie: restituer, d'une part, le patrimoine ethnographique de la Guyane et, d'autre part, marquer sa distance par rapport à la politique d'assimilation dont elle fait l'objet.

11 Lilian PESTRE DE ALMEIDA s'interroge sur la relation oral/écrit à la fois dans l'œuvre de Damas et dans les nouvelles littératures des Amériques, en partant des deux anthologies réalisées par l'écrivain guyanais en 1947 et en 1966. Si la première recense seulement des poètes d'expression française, on relève dans la deuxième une ouverture plus générale à la "poésie du monde noir», au-delà des frontières linguistiques, car toute expression poétique du monde colonisé doit faire face aux problèmes de la relation entre la langue du colonisateur - que ce soit le français, l'anglais, l'espagnol ou le portugais - et les langues du colonisé. Damas devançait déjà, avec ses anthologies, le problème de la catégorisation de ces poètes dans la Négritude, l'Antillanité ou la Créolité, concepts qui font encore aujourd'hui l'objet de discussion critique. La dernière partie de cette étude ouvre un volet sur la réception de Damas dans les Amériques, en distinguant pour ce faire les différentes zones et langues du continent. Si la zone lusophone et hispanophone se montrent moins marquées par l'œuvre damassienne, du fait d'une tradition de poésie nègre déjà solide dans l'espace continental ibéroaméricain, aux États-Unis son audience est réelle mais restreinte à un public d'intellectuels ou d'universitaires, tandis que dans la Caraïbe on le considère un précurseur à la fois de la Négritude et de l'Antillanité.

12 Emmanuel LÉzY ouvre la quatrième section du volume, «De part et d'autre», avec un long article consacré à la relation de Damas avec sa Guyane natale De la Guyane blanche à la Guyane noire, l'éternel retour de Léon Damas. En géographe averti, le chercheur s'interroge sur le regard que Damas porte à la Guyane, lieu de retour, plutôt que de résidence, lieu en noir et blanc où deux univers coexistent: l'un est terrestre, insulaire, orienté vers son propre centre, l'autre est océanique, ouvert. Le premier est lié au mythe édénique de l'Indien doré, le second à l'héritage historique de l'esclavage. Si l'essai Retour de Guyane, écrit par Damas à la suite de la mission ethnologique qui lui fait accomplir un voyage de retour dans son pays natal, présente bien l'univers noir de 
l'esclavage, la Guyane du colonisateur, Veillées noires décrit un pays qui en est l'inverse exact, la grande Guyane insulaire construite à l'image du paradis terrestre.

Antonella EMINA enchaîne son étude à celle d'Emmanuel Lézy, en continuant à s'interroger sur les lieux dans l'œuvre de Damas, à travers un recensement des occurrences des adverbes ici, là ou là-bas, voici et voilà pour essayer d'en tirer la signification profonde. On relève des 245 occurrences du corpus que les adverbes de lieu, loin d'établir un point ferme, restent fluctuants et restituent plutôt un sentiment d'étrangeté globale postcoloniale et contemporaine qui font la modernité, voire la postmodernité de l'écriture damassienne.

Biringanine NDAGANo revient au sujet de l'engagement politique de Damas dans son étude Damas était-il un homme politique?. À travers l'analyse de Retour de Guyane, compte rendu de mission et écrit politique à la fois, l'auteur dégage les idées dont Damas voulait se faire le porte-parole auprès des Français mais qui n'ont pas trouvé une véritable audience. Les écrits journalistiques qu'il rédige après 1938 n'apportent pas non plus d'éclairage nouveau à sa pensée mais continuent à se caractériser par leur virulence. Il faut attendre Misère noire, rédigé en 1939, pour découvrir un autre Damas qui, laissant de côté les tons violents, se fait porteur d'une pensée constructive: l'Europe et les colonies ne représentent plus deux pôles opposés par l'incommunicabilité mais deux réalités qui doivent marcher la main dans la main.

Femi OJO-ADE clôt le volume avec un long article consacré au Rapport Damas, établi par l'écrivain lors des 45 séances de la Commission spéciale d'enquête nommée par le Parlement français pour éclaircir les incidents survenus en Côte d'Ivoire dans les années 1949 et 1950. Bien que ce rapport n'ait jamais été publié, il constitue une source féconde d'information pour les chercheurs engagés dans les études africaines et on y relève des traits qui éclairent la personnalité aux multiples facettes de Damas, à la fois polémiste acéré et politicien contradictoire à l'égard de l'Afrique et des relations interraciales.

La bibliographie des ouvrages utilisés et l'index des noms qui complètent le volume constituent un apparat strictement fonctionnel. Le livre, riche en suggestions, peut être considéré comme la première étape d'une recherche qui mériterait beaucoup d'approfondissements. 